\title{
Absence of significant cross-correlation between WMAP and SDSS (Research Note)
}

\author{
M. López-Corredoira ${ }^{1,2}$, F. Sylos Labini ${ }^{3,4}$, and J. Betancort-Rijo ${ }^{1,2}$ \\ ${ }^{1}$ Instituto de Astrofísica de Canarias, 38200 La Laguna, Tenerife, Spain \\ e-mail: martinlc@iac.es \\ 2 Departamento de Astrofísica, Universidad de La Laguna, 38205 La Laguna, Tenerife, Spain \\ 3 Centro Studi e Ricerche Enrico Fermi, via Panisperna 89 A, Compendio del Viminale, 00184 Rome, Italy \\ 4 Istituto dei Sistemi Complessi CNR, via dei Taurini 19, 00185 Rome, Italy
}

Received 25 June 2009 / Accepted 17 January 2010

ABSTRACT

\begin{abstract}
Aims. Several authors have claimed to detect a significant cross-correlation between microwave WMAP anisotropies and the SDSS galaxy distribution. We repeat these analyses to determine the different cross-correlation uncertainties caused by re-sampling errors and field-to-field fluctuations. The first type of error concerns overlapping sky regions, while the second type concerns nonoverlapping sky regions.

Methods. To measure the re-sampling errors, we use bootstrap and jack-knife techniques. For the field-to-field fluctuations, we use three methods: 1) evaluation of the dispersion in the cross-correlation when correlating separated regions of WMAP with the original region of SDSS; 2) use of mock Monte Carlo WMAP maps; 3) a new method (developed in this article), which measures the error as a function of the integral of the product of the self-correlations for each map.

Results. The average cross-correlation for $b>30 \mathrm{deg}$ is significantly stronger than the re-sampling errors - both the jack-knife and bootstrap techniques provide similar results - but it is of the order of the field-to-field fluctuations. This is confirmed by the crosscorrelation between anisotropies and galaxies in more than the half of the sample being null within re-sampling errors.

Conclusions. Re-sampling methods underestimate the errors. Field-to-field fluctuations dominate the detected signals. The ratio of signal to re-sampling errors is larger than unity in a way that strongly depends on the selected sky region. We therefore conclude that there is no evidence yet of a significant detection of the integrated Sachs-Wolfe (ISW) effect. Hence, the value of $\Omega_{\Lambda} \approx 0.8$ obtained by the authors who assumed they were observing the ISW effect would appear to have originated from noise analysis.
\end{abstract}

Key words. cosmic microwave background - large-scale structure of Universe

\section{Introduction}

Several authors (Fosalba et al. 2003; Vielva et al. 2006; Cabré et al. 2006; Raccanelli et al. 2008; Ho et al. 2008; Granett et al. 2008) have claimed that there is a significant cross-correlation between cosmic microwave background radiation (CMBR) anisotropies and the density of galaxies, which is interpreted as the integrated Sachs-Wolfe (ISW) effect. An anticorrelation caused by the Sunyaev-Zel'dovich effect would also be expected on scales smaller than $\sim 1^{\circ}$, but this is negligible when averaging large regions of the sky (Hernández-Monteagudo \& Rubiño-Martín 2004). The conclusion of these authors is that the measured cross-correlation should be interpreted as a detection of the ISW effect within a $\Lambda \mathrm{CDM}$-cosmology and it serves to constrain the value of the cosmological parameters.

We reanalyze whether this correlation exists by considering galaxies observed by the Sloan Digital Sky Survey (SDSS), taking particular care in the calculation of the cross-correlation errors. The root mean square (rms) of the cross-correlation for distant, widely different areas of sky (here called "field-tofield" errors) infer much larger errors than those calculated using re-sampling cross-correlations techniques i.e., when these are determined in different strongly overlapping and thus not independent subsamples of a given sample (re-sampling errors). We conclude that measurements of the errors in the crosscorrelation function for overlapping sub-fields lead to an underestimate of the true scatter in the signal.

\section{Data}

We consider two types of data for the two fields that we crosscorrelate:

1. Microwave temperature anisotropies $(\delta T)$ from the 5 th year WMAP release (Hinshaw et al. 2009). We use the $V$-band $(61 \mathrm{GHz})$ data because of its lower level of pixel noise. We checked that the results of this paper are approximately similar if we use the W-band $(94 \mathrm{GHz}$ ) data. There is no need to subtract foreground Galactic contamination because this is not correlated with galaxy counts (corrected for extinction), and because this is small in off-plane regions. In any case, the published foreground corrections might not be enough accurate (López-Corredoira 2007). We assign the same weight to each WMAP pixel of equal size.

2. Galaxy counts $(G)$ are obtained from the survey SDSS, photometric catalog, data release DR7 (Abazajian et al. 2009). They cover an area $11663 \mathrm{deg}^{2}$ (28\% of the sky) mostly in the northern Galactic hemisphere. We did not use the striped region data with $b<30^{\circ}$ to ensure low Galactic extinction and avoided negative latitudes because these are small isolated regions dominated by edge effects. We used only galaxies with $r$ magnitudes in the range [18,21] (Galacticextinction corrected) (within these limits galaxy counts are complete) and "clean photometry" according to an SDSS algorithm (e.g., we removed sources close to saturated objects with contamination of their by other objects), and avoiding 
the borders by $\sim 0.3 \mathrm{deg}$. In this situation, the total used area is $7349 \mathrm{deg}^{2}$ (18\% of the sky), containing 2.2, 6.4 and 17.1 million galaxies in the $r$ magnitude ranges $[18,19],[19,20]$, and [20,21], respectively.

\section{Methods}

By defining the galaxy count $(G)$ density contrast to be $\delta_{G}(\theta)=$ $(G(\theta)-\langle G\rangle) /\langle G\rangle$, and denoting by $\delta_{T}(\theta)=T(\theta)-T_{0}$ the fluctuations in the CMBR with respect to the average temperature $T_{0}$, the cross-correlation function can be written as

$\omega_{T G}(\theta) \equiv\left\langle\delta_{T}(\theta) \delta_{G}(0)\right\rangle$.

The estimator of Eq. (1) computes the cross-correlation to be the average over all pixels with separations $\theta \pm(\Delta \theta / 2)$, where $\Delta \theta$ is the step between successive values of $\theta$. In what follows, we set $\Delta \theta \approx 0.29 \mathrm{deg}$.

There are two kinds of errors in the cross-correlation, associated with two distinct ways of constructing sub-fields over which they are computed (Sylos Labini et al. 2009):

1. Re-sampling errors: for point distributions, there is a component of the total error that is caused by the finiteness of the number of points and is closely related to that given by the resampling techniques (Betancort-Rijo 1991); however, here, in the correlation of two continuous fields, the association is not at all clear. These may be estimated with a re-sampling technique, for instance jack-knife or bootstrap. In the latter case, we calculate $n_{\mathrm{s}}$ times the cross-correlation by removing each time a different fraction $1 / n_{\mathrm{s}}$ of the $N$ pixels. By using the bootstrap method, we also calculate a number $n_{\mathrm{s}}$ of times the cross-correlation that each time chooses the same number $N$ of pixels from the original sample, but randomly selected (so that there are some pixels that are selected several times, while others are not selected at all). Both in bootstrap and jack-knife, we then calculate the rms of these $n_{\mathrm{s}}$ resamplings, which provides our error. We use $n_{\mathrm{s}}=10$, which implies that the relative error in the rms is $\left(2 n_{\mathrm{s}}\right)^{-1 / 2} \sim 20 \%$ for Gaussian errors. We note that for both techniques the $n_{\mathrm{s}}$ determination have been performed on overlapping subsamples, and they are thus not independent.

2. Field-to-field fluctuations: these are caused by intrinsic fluctuations in both the large-scale structure of galaxies and the microwave temperature field. We propose three methods for estimating these fluctuations:

(a) Different fields: We cross-correlate the $G$ field in the full area with a different field $\delta T_{*}$ of the same power spectrum as the original WMAP data, although uncorrelated with $G$. One simple way of applying this method is assigning to $\delta T_{*}$ the value of its own WMAP data but in other regions of the sky that are completely separated. For instance, we define $\delta T_{*}(l, b)=$ $\delta T(l+\beta,-b)$ with different values of $\beta$ (we consider $n_{\mathrm{s}}=10$ different values: $\beta=2 i \pi / n_{\mathrm{s}}, i=1$ to $n_{\mathrm{s}}$ ), and calculate the rms for the $n_{\mathrm{s}}$ realizations. In this case, we use a small enough number $\left(n_{\mathrm{s}}\right)$ of regions, so the relative error in the rms is $\left(2 n_{\mathrm{S}}\right)^{-1 / 2} \sim 20 \%$ for Gaussian errors. The cross-correlations at scales 60 $180^{\circ}$ might produce some signal, but this would be small, given that the self-correlation of $\delta T$ is almost zero for $\theta>60^{\circ}$ (Copi et al. 2009). The possible large-scale cross-correlations of the different fields infer that this estimation of the rms value is a conservative upper-limit value.

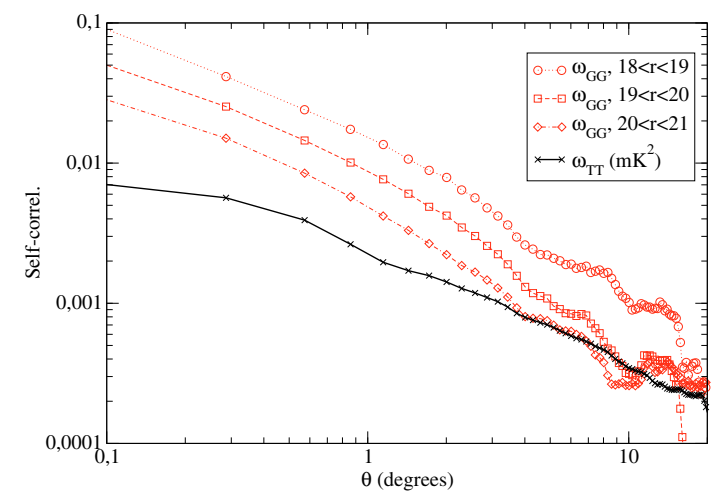

Fig. 1. Log-log of the self-correlations of the fields $\delta_{G}$ and $\delta_{T}$.

(b) Monte Carlo simulations of WMAP: We generate a number of Monte Carlo realizations of WMAP by using the software "synfast" to generate random mock maps of anisotropies corresponding to the theoretical power spectrum (Hinshaw et al. 2009) filtered for the V-band. We perform $n_{\mathrm{s}}=100$ realizations, and then calculate the rms of their cross-correlation with the fixed SDSS galaxy counts map. The relative error in the rms is $\left(2 n_{\mathrm{s}}\right)^{-1 / 2}=$ $7 \%$.

(c) Integral of the self-correlations: A calculation of the field-to-field variance in the cross-correlation of two non-correlated fields can be given by (see Appendix A)

$\sigma_{\omega_{T G}}^{2}(\theta)=2\left\langle\omega_{T T}\left(\theta_{1,2}\right) \omega_{G G}\left(\theta_{3,4}\right)\right\rangle_{4, \theta}$,

where $\langle(\ldots)\rangle_{4, \theta}$ stands for the average extended over all groups of four pixels $(1,2,3,4)$ in a region in which the separation between pixels 1,3 and 2,4 is between $\theta-\Delta \theta / 2$ and $\theta+\Delta \theta / 2, \theta_{1,2}$ is the separation of pixels $1,2, \theta_{3,4}$ is the separation of pixels 3,4 , and $\omega_{T T}$ and $\omega_{G G}$ are the self-correlations, respectively, for the fields $\delta_{T}$ and $\delta_{G}$. We note that with this method we assume that $\delta_{T}$ and $\delta_{G}$ are uncorrelated (as in Monte Carlo simulations); therefore, $\sigma$ refers to the limits of pure non-correlated fields within the corresponding probabilities (68\%). In addition, we note that we use the self-correlations that we measure in our fields (see Fig. 1), i.e., we have only one realization. Cosmic variance would introduce some extra uncertainty.

\section{Results}

In Fig. 1, we plot the self-correlations. In Fig. 2, we show the determination of the cross-correlation function for different ranges of magnitude, and the errors computed by using re-sampling errors and field-to-field determinations. On the one hand, the errors computed by both the bootstrap and the jack-knife method are of the same order, and on the other hand the three "fieldto-field" methods yield similar results, which however are much larger than the re-sampling errors. The "Different fields" method yields in general a slightly lower rms than the integral of the self-correlations, possibly because of small positive large-scale correlations, which slightly reduce the dispersion, as mentioned in discussing "different fields" in Sect. 3. The "Monte Carlo" method might yield slightly higher values of rms than the integral of the self-correlations due to the larger amplitude of the low-multipoles in the theoretical power spectrum.

The field-to-field fluctuations obtained by using independent determinations of the cross-correlation function are similar to the amplitude of the detected signal or even larger. Figure 3 

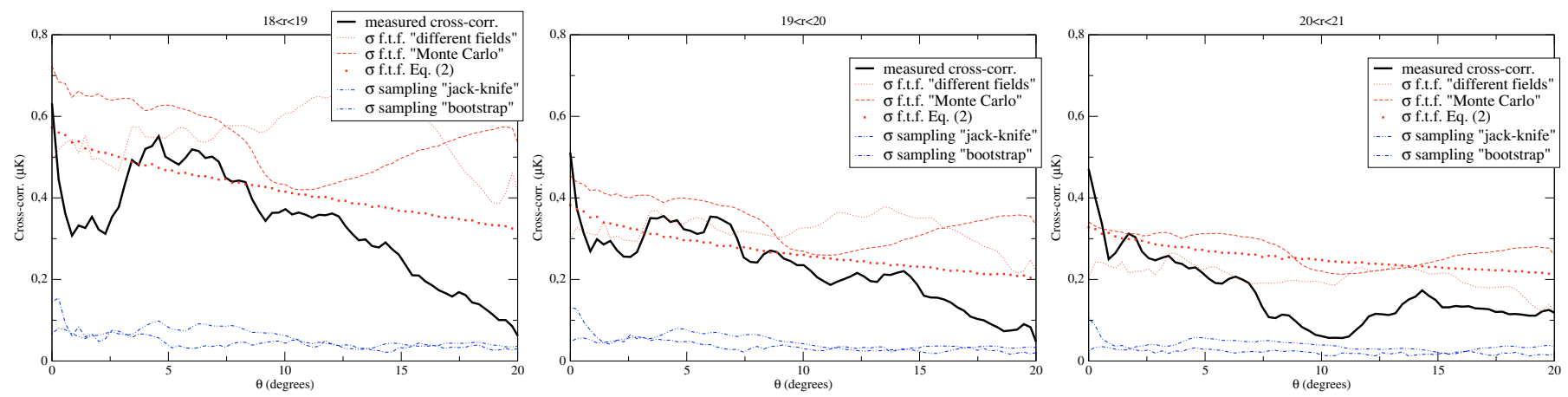

Fig. 2. Cross-correlation function $\omega_{T G}$ WMAP-SDSS (black line) for galaxies with $b<30^{\circ}$ and in the magnitude range $18<r<19$ (left panel), $19<r<20$ (center panel), and $20<r<21$ (right panel). The rms value calculated by resampling errors and field-to-field fluctuations are also plotted.

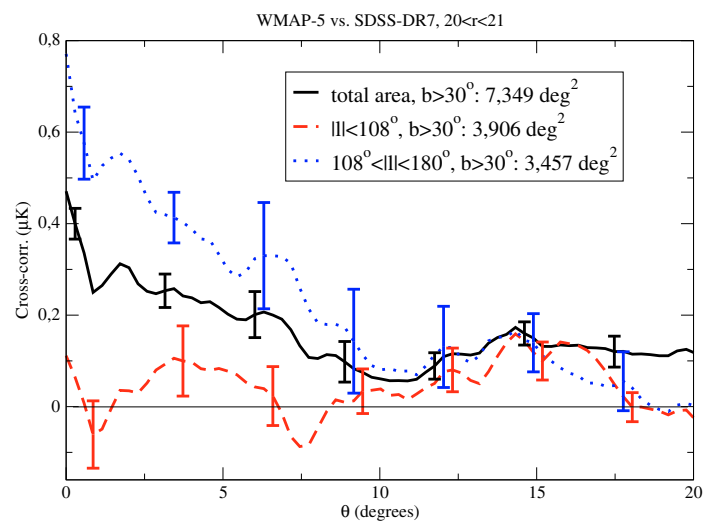

Fig. 3. Cross-correlation $\left(\omega_{T G}\right)$ WMAP-SDSS, $20<r<21$ : the average of the whole selected SDSS-DR7 area, and the average for $|l|<108^{\circ}$ and $108^{\circ}<|l|<180^{\circ}$; error bars represent jack-knife re-sampling errors.

illustrates this point by showing that there are no positive average cross-correlations in a sky region of area more than half of the full angular coverage.

From all these analyses, we cannot exclude the value of $\left|\omega_{T G}\right|$ being compatible with zero for any $\theta$ within field-to-field fluctuations. Thus we conclude that there is no significant crosscorrelation detection. This situation is similar to that found for the SDSS 3D self-correlation by Sylos-Labini et al. (2009), who also demonstrated that the field-to-field fluctuations are of the order of the signal in the previously announced discovery of baryon acoustic oscillations and large-scale anticorrelations.

\section{Comparison with previous works}

Other authors who calculated the cross-correlation between WMAP and SDSS galaxy counts measured a significant signal. For instance, Cabré et al. (2006) measured a value of $\omega_{T G}\left(3^{\circ}\right) \sim$ $0.5 \mu \mathrm{K}$ and a significant positive $\omega_{T G}$ for all angles lower than 20 degrees for the subsample $20<r<21$ over 5500 square degrees of SDSS-DR4. Giannantonio et al. (2008) obtained a value of $\omega_{T G}\left(3^{\circ}\right) \sim 0.3 \mu \mathrm{K}$ for the subsample $18<r<21$ of SDSS-DR6, excluding the southern Galactic hemisphere and high Galactic extinction regions. In addition, they found a significant positive signal out to $\theta=8$ degrees. Their values are more or less compatible with our estimate of the cross-correlation function, within the re-sampling error bars and taking into account that their subsamples are slightly different. However we do not measure significant cross-correlations, whereas these authors do a result we cannot explain.

Cabré et al. (2006) and Giannantonio et al. (2008) performed Monte Carlo simulations using mock maps, and obtained similar values or ones only slightly larger than a jack-knife. We do not know whether these disagreement are caused by mistakes in their calculations or whether their claim is that re-sampling errors represent the full errors. Other authors used only jackknife technique errors (e.g., Sawangwit et al. 2009). A similar problem may affect the results of Raccanelli et al. (2008), who measured the cross-correlation between NVSS radio sources and WMAP anisotropies. Raccanelli et al. (2008) calculate the error in simulating 1000 mock NVSS maps by randomly distributing the unmasked pixels of the true NVSS maps. We are concerned that this process might destroy part of the self-correlation of each map, and that the errors might not represent the full field-to-field fluctuations. There has been considerable discussion of these errors (Cabré et al. 2007; Giannantonio et al. 2008). However, against their claims one can infer from the analyses of this paper that: i) jack-knife or bootstrap methods do not calculate the whole error; ii) the level field-to-field fluctuations is as large as the measured average signal. In addition, our conclusion is that the signal is largely dependent on the specific sub-region chosen. We find that in the large area of $|l|<108^{\circ}, b>30^{\circ}$ (3906 square degrees available with SDSS-DR7, more than half of the sample) we do not measure any signal, so the average signal of the entire sample must be caused by a fluctuation.

One remarkable aspect of the analysis of WMAP/SDSSDR4 is that Cabré et al. (2006) obtain a $3.6 \sigma$ detection for $20<r<21$, while Giannantonio et al. (2008) with a wider areal coverage (SDSS-DR6) and broader range of magnitudes, $18<$ $r<21$, achieve only a $2.3 \sigma$ detection. This decrease in the significance is unexpected if the signal were real. We also note that some authors calculated the combined signal to noise ratio of different cross-correlations in different samples, obtaining values over $4 \sigma$, by summing them quadratically (Cabré et al. 2006). This is incorrect because they do not take into account the correlation between the samples, thus neglect an important part of the estimated error.

A higher significance in the cross-correlation of WMAP/SDSS is claimed to be obtained (Granett et al. 2008) when only super-clusters/super-voids are correlated with WMAP instead of the entire SDSS survey: a value of $4.4 \sigma$. Apart from our questions raised above, we are also concerned about possible a posteriori fitted parameters used to obtain this correlation. For instance, Granett et al. (2008) separate regions of the sky centered on super-clusters with radii of 4 degrees; we 
ask why 4 degrees? These authors illustrate that the significance is only $3.5 \sigma$ for radius $3 \mathrm{deg}$ or $3.8 \sigma$ for $5 \mathrm{deg}$. The significance also changes with the number of superclusters/supervoids selected, being $4.4 \sigma$ with $N=50$ but only $2.8 \sigma$ with $N=70$. A signal to noise ratio of $2-3 \sigma$ is provided by other authors without any selection of super-clusters, so they appear to have considered both a radius and number of superclusters/supervoids that achieves the maximum increase in the signal to noise ratio (from 2-3 up to to 4.4).

We note that Bielby et al. (2010) measured the correlation of WMAP anisotropies with emission-line galaxies selected photometrically from SDSS and inferred a non-significant correlation, with large field-to-field errors comparable to those we obtain (we are cautious in interpreting their result, however, because the cross-correlation in the different subfields are not independent and this affects the way in which they have been using to determine the rms). They claim that their result implies that possibly emission line galaxies are more strongly clustered and less correlated with microwave anisotropies, something that is not entirely clear to us. In our opinion, the results of Bielby et al. (2010) of non-significant cross-correlation may be correct and there is unlikely to be a difference in the interaction of galaxies with the background CMBR that is caused entirely by them having emission lines. Sawangwit et al. (2010) failed to measure a significant cross-correlation between the luminous red galaxies of SDSS-DR5 and WMAP. On the other hand, they found some positive correlation of WMAP with 2SLAQ survey, and negative correlation of WMAP with the AAOmega survey. Analyses by Hernández-Monteagudo (2008) demonstrated that the crosscorrelation of WMAP/SDSS-DR4 should have at least within a signal/noise ratio of $0.7-1.7$, much lower than the significance obtained by the authors cited above. These results might be interpreted as independent confirmations of our results here.

Apart from those analyzing data from SDSS, previous studies reach the general conclusion that the ISW effect was not detected significantly in: (1) cross-correlations with X-ray XRB, Boughn \& Crittenden (2003) claiming an absence of the ISW using X-ray data; (2) near-infrared 2MASS, Francis \& Peacock (2009) not finding any corresponding ISW signal; or (3) radio sources NVSS, Hernández-Monteagudo (2009) casting doubt on the correlation between WMAP and NVSS radio sources, since the cumulative signal to noise ratio of the cross-correlation with multipoles $l<60$ is lower than 1, and the ISW itself, since the signal to noise ratio should be around 5 theoretically.

\section{Conclusions}

We concluded that there is no significant cross-correlation between the CMBR temperature anisotropies of WMAP and the galaxy counts of the SDSS, and any claims to have detected the ISW effect on the basis of significant cross-correlation are unjustified. Field-to-field fluctuations dominate the detected signals. Any detection of signal is very dependent on the selected region of the sky. Other authors erroneously claimed to have detect significant correlations because they had used a particular sky region with a fluctuation that is not representative of the average sky or because they had underestimated the statistical errors by using non-independent re-samplings. If our conclusion is correct, the value of $\Omega_{\Lambda} \approx 0.8$ obtained by those authors based on the assumption of observing the ISW effect would have been one induced by noise. Its value would be coincident with the expected value for $\Lambda \mathrm{CDM}$ by chance, and in the spirit of accepting a scientific result when it indeed produces numbers expected a priori.

Acknowledgements. We thank an anonymous referee for useful comments and suggestions. Thanks are given to J. A. Rubiño-Martín and R. Génova-Santos from IAC (Tenerife) for helpful comments and help in the use of HEALPIX and SYNFAST software. Thanks are given to Claire Halliday (language editor of A\&A) for proof-reading of the text. Funding for the SDSS has been provided by the Alfred P. Sloan Foundation, the Participating Institutions, the National Science Foundation, the US Department of Energy, the NASA, the Japanese Monbukagakusho, the Max Planck Society, and the Higher Education Funding Council for England. The SDSS Web Site is: http.//wWw . sdss.org/. WMAP is the result of a partnership between Princeton University and NASA's Goddard Space Flight Center. MLC was supported by the Ramón y Cajal Programme of the Spanish Science Ministry.

\section{Appendix A: Field-to-field errors in the cross-correlation as an integral of the self-correlations for two uncorrelated fields ${ }^{1}$}

We consider two continuous random scalar fields, $F_{A}$ and $F_{B}$, in a space with $d$ dimensions and any topology. Without loss of generality, we shall assume that the mean values (over realization) of both fields is zero and they are not correlated: $\left\langle F_{A}\right\rangle=\left\langle F_{B}\right\rangle=\left\langle F_{A} F_{B}\right\rangle=0$. On the other hand, the values of each field at two different points $\boldsymbol{x}_{1}, \boldsymbol{x}_{2}$ are not independent random variables: $\omega_{A / B}\left(\boldsymbol{x}_{1}, \boldsymbol{x}_{2}\right)=\left\langle F_{A / B}\left(\boldsymbol{x}_{1}\right) F_{A / B}\left(\boldsymbol{x}_{2}\right)\right\rangle$, where the average is over realizations. In practice, in most interesting cases the fields are statistically homogeneous and ergodic, so that $\omega$ depends only on $\boldsymbol{x}_{1}-\boldsymbol{x}_{2}$, and the correlation may be defined as spatial averages, which is the useful definition since in most cases only one realization is available. If the fields are also statistically isotropic, $\omega$ depends only on $r \equiv\left|\boldsymbol{x}_{1}-\boldsymbol{x}_{2}\right|$. For the following derivation, we shall assume homogeneity and isotropy; the full expression might easily be recovered if needed.

We first derive the "field-to-field error" (i.e., the true error) for the zero lag estimator:

$E\left[\omega_{A B}(r=0)\right]=\frac{1}{N} \sum_{i=1}^{N} F_{A}(i) F_{B}(i)$,

where $F_{A / B}(i) \equiv F_{A / B}\left(\boldsymbol{x}_{i}\right)$. We have replaced the $d$-dimensional volume integral over the sample with a sum over $N$ equal volume cell indexed by $i$ and centered on $\boldsymbol{x}_{i}$. We would have to multiply the contribution of the field for each pixel by a weight equal to the volume of the pixel in the case of non-equal volume cells.

For the variance in Eq. (A.1), we have:

$\sigma_{\omega_{A B}}^{2}(r=0)=\left\langle E^{2}\right\rangle-\langle E\rangle^{2}=\left\langle E^{2}\right\rangle$,

since, by construction, the mean value of $E$ over realizations, $\omega_{A B}$, is assumed to be zero. Developing the square of expression (A.1), and taking its average, we have:

$\left\langle E^{2}\right\rangle=\frac{2}{N^{2}}\left\langle\sum_{i, j}^{N} F_{A}(i) F_{B}(i) F_{A}(j) F_{B}(j)\right\rangle$.

In principle, the factor of 2 should not be there in the case $i=j$, but this will be negligible in the limit of arbitrarily small cells.

Now, since the fields $F_{A}$ and $F_{B}$ are uncorrelated

$$
\begin{aligned}
\left\langle F_{A}(i) F_{B}(i) F_{A}(j) F_{B}(j)\right\rangle & =\left\langle F_{A}(i) F_{A}(j)\right\rangle\left\langle F_{B}(i) F_{B}(j)\right\rangle \\
& =\omega_{A}\left(r_{i j}\right) \omega_{B}\left(r_{i j}\right),
\end{aligned}
$$

1 By J. Betancort-Rijo. 
where $r_{i j} \equiv\left|\boldsymbol{x}_{i}-\boldsymbol{x}_{j}\right|$. Thus, we have

$$
\begin{aligned}
\sigma_{\omega_{A B}}^{2}(r=0) & =2\left\langle\omega_{A}(r) \omega_{B}(r)\right\rangle \\
& \equiv \frac{2}{V_{\mathrm{s}}^{2}} \iint_{\text {sample }} \omega_{A}\left(r_{12}\right) \omega_{B}\left(r_{12}\right) \mathrm{d}^{d} \boldsymbol{r}_{1} \mathrm{~d}^{d} \boldsymbol{r}_{2},
\end{aligned}
$$

where $V_{\mathrm{s}}$ represents the volume of the sample.

The correlation estimator for any non-zero lag is

$E\left[\omega_{A B}\left(r_{0}\right)\right]=\frac{1}{N^{2}} \sum_{i, j / r_{i j}=r}^{N} F_{A}(i) F_{B}(j)$,

where $r_{0}-\Delta r / 2<r<r_{0}+\Delta r / 2$. Following Eq. (A.6) using the same procedure as for Eq. (A.1), one obtains

$\sigma_{\omega_{A B}}^{2}\left(r_{0}\right)=2\left\langle\omega_{A}\left(r_{12}\right) \omega_{B}\left(r_{34}\right)\right\rangle_{4, r_{0}} \equiv$

$\frac{2 \iint_{\text {sample } / r_{13}=r, r_{24}=r} \omega_{A}\left(r_{12}\right) \omega_{B}\left(r_{34}\right) \mathrm{d}^{d} \boldsymbol{r}_{1} \mathrm{~d}^{d} \boldsymbol{r}_{2} \mathrm{~d}^{d} \boldsymbol{r}_{3} \mathrm{~d}^{d} \boldsymbol{r}_{4}}{\iint_{\text {sample } / r_{13}=r, r_{24}=r} \mathrm{~d}^{d} \boldsymbol{r}_{1} \mathrm{~d}^{d} \boldsymbol{r}_{2} \mathrm{~d}^{d} \boldsymbol{r}_{3} \mathrm{~d}^{d} \boldsymbol{r}_{4}}$.

Equation (A.7), and its particular case, Eq. (A.5), infer the variance over realizations of the estimator of the correlation between two uncorrelated fields $A, B$ when any new global realization of both fields is carried out. In the case when we fix the realization of one of the fields while changing the other, Eq. (A.7) is also valid but the self-correlation of the fixed realization must be calculated by averaging over pixels in this fixed realization, rather than over realizations. Now, since the estimated self-correlation may fluctuate above and below the universal (mean of all realizations) value, it is clear that the variance in the estimator of the cross-correlation of $A$ and $B$ when one of them is kept fixed may be slightly above or below the one corresponding to the case when both fields fluctuate.

\section{References}

Abazajian, K. N., Adelman-McCarthy, J. K., Agüeros, M. A., et al. 2009, ApJS, 182,543

Betancort-Rijo, J. E. 1991, A\&A, 245, 347

Bielby, R., Shanks, T., Sawangwit, U. et al. 2010, MNRAS, 403, 1261

Boughn, S. P., \& Crittenden, R. G. 2003, in: The emergence of Cosmic Structure, AIP Conf. Proc., 666, 67

Cabré, A., Gaztañaga, E., Manera, M., Fosalba, P., \& Castander, F. 2006, MNRAS, 372, L23

Cabré, A., Fosalba, P., Gaztañaga, E., \& Manera, M. 2007, MNRAS, 381, 1347

Copi, C. J., Huterer, D., \& Starkman, G. D. 2009, MNRAS, 399, 295

Francis, C. L., \& Peacock, J. A. 2009, [arXiv:0909.2494]

Fosalba, P., Gaztañaga, E., \& Castander, F. J. 2003, ApJ, 597, L89

Giannantonio, T., Scranton, R., Crittenden, R. G. et al. 2008, Phys. Rev. D, 77, 3520

Granett, B. R., Neyrinck, M. C., \& Szapudi, I. 2008, ApJ, 683, L99

Hernández-Monteagudo, C. 2008, A\&A, 490, 15

Hernández-Monteagudo, C. 2009, [arXiv:0909 .4294]

Hernández-Monteagudo, C., \& Rubiño-Martín, J. A. 2004, MNRAS 347, 403

Hinshaw, G., Weiland, J. L., Hill, R. S., et al. 2009, ApJS, 180, 225

Ho, S., Hirata, C., Padmanabhan, N., Seljak, U., \& Bahcall, N. 2008, Phys. Rev. D, 78, 3519

López-Corredoira, M. 2007, J. Astrophys. Astron. 28, 101

Raccanelli, A., Bonaldi, A., Negrello, M. et al. 2008, MNRAS, 386, 2161

Sawangwit, U., Shanks, T., Cannon, R. D., et al. 2010, MNRAS, 402, 2228

Sylos Labini, F., Vasilyev, N. L., Baryshev, Yu. V., \& López-Corredoira, M. 2009, A\&A, 505, 981

Vielva, P., Martínez-González, E., \& Tucci, M. 2006, MNRAS, 365, 891 